\title{
Sensing Volume of Open-Ended Coaxial Probes for Dielectric Characterization of Breast Tissue at Microwave Frequencies
}

\author{
Dina M. Hagl, Student Member, IEEE, Dijana Popovic, Student Member, IEEE, Susan C. Hagness, Member, IEEE, \\ John H. Booske, Senior Member, IEEE, and Michal Okoniewski, Senior Member, IEEE
}

\begin{abstract}
We are using open-ended coaxial probes to determine the dielectric properties of freshly excised normal and diseased breast tissue specimens. The considerable variability in size and composition of these specimens predicates the need for determining the minimum surgical specimen size that yields accurate measurements for a given probe diameter. We investigate the sensing volume of $2.2-$ and 3.58-mm-diameter flange-free coaxial probes for both low- and high-water-content tissue using standard liquids that exhibit dielectric properties similar to breast tissue over the microwave frequency range from 1 to $20 \mathrm{GHz}$. We also present an innovative graphical technique based on the use of Cole-Cole diagrams to determine the error thresholds in the magnitude and phase of the reflection coefficient, which bound the errors in the measured complex permittivity to an acceptable level. Results from self-consistent experiments and finite-difference time-domain simulations indicate that a tissue specimen with a thickness of $3.0 \mathrm{~mm}$ and a transverse dimension of $1.1 \mathrm{~cm}$ is the minimum size that yields accurate measurements with the 3.58-mm-diameter probe. For the 2.2-mm-diameter probe, the specimen's thickness and width should be at least 1.5 and $5 \mathrm{~mm}$, respectively. These conclusions are relevant not only to breast tissue characterization, but also more generally to the dielectric characterization of a variety of low- and high-water-content biological tissues.
\end{abstract}

Index Terms-Biological materials, coaxial probes, dielectric spectroscopy, microwave measurements.

\section{INTRODUCTION}

$\mathbf{T}$ HE critical need for new technologies to improve detection, diagnosis, and treatment of breast cancer is widely recognized [1]. A number of innovative technologies employing radio waves or microwaves are under investigation for this purpose [2]-[8]. Such efforts have been guided by

Manuscript received June 8, 2002; revised December 10, 2002. This work was supported by The Whitaker Foundation under Biomedical Engineering Research Grant RG-99-0004, by the National Institutes of Health under Grant R01 CA087007-01 awarded by the National Cancer Institute, and by the Natural Sciences and Engineering Research Council of Canada.

D. M. Hagl was with the Department of Electrical and Computer Engineering, University of Wisconsin-Madison, Madison, WI 53706 USA. She is now with the Bundeswehr Universitaet, Munich D-85577, Germany (e-mail: dina@hagl.net).

D. Popovic and M. Okoniewski are with the Department of Electrical Engineering, University of Calgary, Calgary, AB, Canada T2N-1N4 (e-mail: dpopovic@ieee.org; michal@enel.ucalgary.ca).

S. C. Hagness and J. H. Booske are with the Department of Electrical and Computer Engineering, University of Wisconsin-Madison, Madison, WI 53706

USA (e-mail: hagness@engr.wisc.edu; booske@engr.wisc.edu).

Digital Object Identifier 10.1109/TMTT.2003.809626 published data in the literature suggesting that a substantial contrast exists between the dielectric properties of normal and cancerous breast tissue [9]-[12]. However, the existing data is limited both in frequency (below $3 \mathrm{GHz}$ ) and in the extent of investigated tissue types. To expand this data set, the University of Wisconsin-Madison (UW) and the University of Calgary (UC), are collaborating to conduct a comprehensive set of measurements of the dielectric properties of normal, benign, and malignant breast tissue at microwave frequencies. The comprehensive dielectric-properties database resulting from these measurements on freshly excised breast biopsy, mastectomy, lumpectomy, and reduction specimens will further facilitate the engineering of noninvasive nonionizing microwave technology to complement and augment early detection, diagnostic, and treatment technologies currently employed in the detection and management of breast cancer.

Open-ended coaxial probes have been widely used to measure the dielectric properties of biological tissues over a broad range of radio and microwave frequencies. Since the coaxial probe technique does not require tissue manipulation or preparation, dielectric-properties measurements can be integrated in a straightforward manner with surgical and pathology protocols. Consequently, in vivo measurements are feasible and reliable ex vivo measurements are achievable using open-ended coaxial probes. The end of the probe is simply placed in contact with the tissue sample and the complex input reflection coefficient is measured as a function of frequency using a vector network analyzer (VNA). The measured reflection coefficient is then converted to a complex permittivity using one of a number of analytical or numerical models (e.g., see [13]-[18]).

Accurate dielectric characterization of excised breast tissue ${ }^{1}$ using an open-ended coaxial probe requires the sample to be homogeneous within a volume large enough so that its measured reflection coefficient is identical to that of a sample filling an entire half-space. Excised breast tissue specimens vary considerably in physical size, ranging from excisional biopsy specimens as small as $1 \mathrm{~cm}$ in diameter to breast reduction specimens as large as $10 \mathrm{~cm}$ or more in diameter. The composition of tissue within a sample may also vary. For example, breast reduction specimens contain both regions of concentrated fibroglandular tissue and regions of fatty tissue,

\footnotetext{
${ }^{1}$ Here, we focus our discussion on ex vivo measurements involving specimens that are small in size and/or heterogeneous in composition. We note, however, that the issues discussed in this paper are also relevant to in vivo measurements of heterogeneous tissue.
} 
and normal tissue is often found around the periphery of malignant or benign tissue present in biopsy, mastectomy, and lumpectomy samples. Furthermore, breast tissue specimens vary considerably in their dielectric properties. For example, based on published data [9]-[12] and our own preliminary data, the dielectric constant of breast tissue ranges between approximately 5-60, depending on the tissue type and pathology. If the sample is electrically small relative to the probe aperture size, reflections at the edges may perturb the measured reflection coefficient. A small homogeneous region within an otherwise heterogeneous tissue sample poses similar problems. While recently developed models of open-ended probes can account for the finite thickness of the test sample, the high degree of variability in both thickness and transverse extent of breast tissue specimens makes it impractical to try to apply such models. The strategy we have adopted has been to develop a set of exclusion criteria that indicates when a surgical specimen (or region of homogeneous tissue within a larger specimen) is too small to yield accurate measurements for a given probe aperture size. Development of accurate criteria requires a careful analysis of the extent of penetration of the near fields in the axial and transverse directions for a narrow-diameter coaxial probe-a characteristic we refer to as the sensing volume of the probe.

Guidelines for choosing the appropriate probe size in relation to the electrical properties of a sample of infinite size have been presented in a number of papers (see, e.g., [13], [14], and [19]). A limited number of studies to date have considered the effect of a finite sample size on the measurement accuracy when an infinite sample is assumed in the conversion from reflection coefficient to permittivity. For example, finite-difference time-domain (FDTD) simulations of a flanged air-filled coaxial probe were used in [20] to numerically investigate the influence of the transverse dimension of a low-loss solid sample (Teflon) on the reflection coefficient. Experimental measurements were conducted in [21] using a flanged Teflon-filled coaxial probe to investigate the influence of the depth dimension of a low-loss solid sample (paper) on the accuracy of the measured permittivity. In [22], both FDTD simulations and experimental measurements of the sensing volume were conducted at a single frequency $(860 \mathrm{MHz})$ to quantify the sensing volume of a flanged largediameter Teflon-filled coaxial probe immersed in a high-loss liquid sample (saline).

The purpose of the comprehensive study presented in this paper is to determine the sensing volume of narrow-diameter coaxial probes as a function of probe aperture size, frequency, and breast tissue permittivity. Our study is based on both FDTD simulations and experimental measurements. We investigate narrow-diameter coaxial probes without flanges since they are most suitable for characterizing breast tissue at microwave frequencies. In Section II, we discuss our methodology for quantifying the sensing volume. Section III presents our raw data and processed results, including comparisons between UW and UC results and between experimental and FDTD-computed data. We conclude with a set of guidelines for the minimum allowable breast tissue specimen size needed to achieve the desired level of accuracy in the measured dielectric properties.

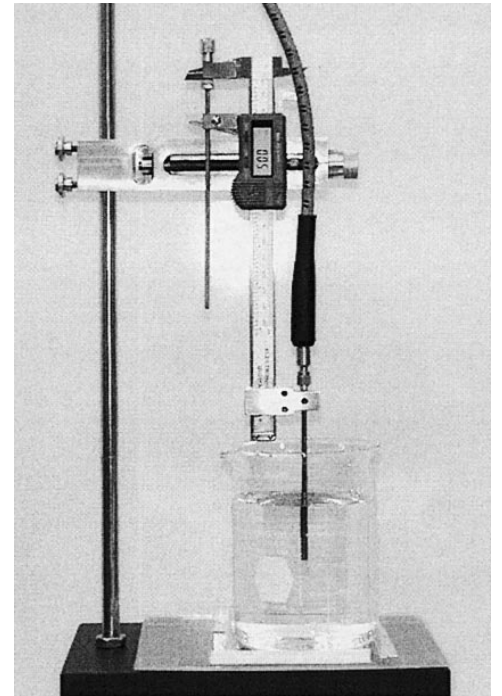

Fig. 1. Experimental setup for measuring the sensing volume of an open-ended coaxial probe.

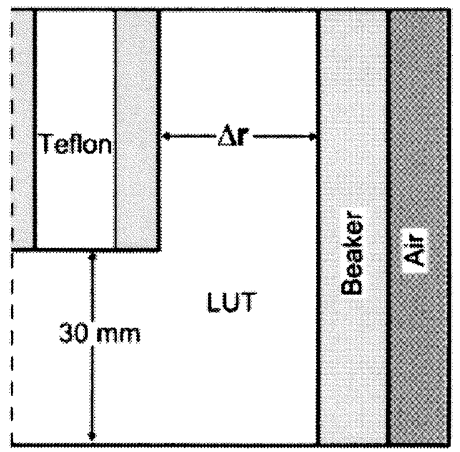

(a)

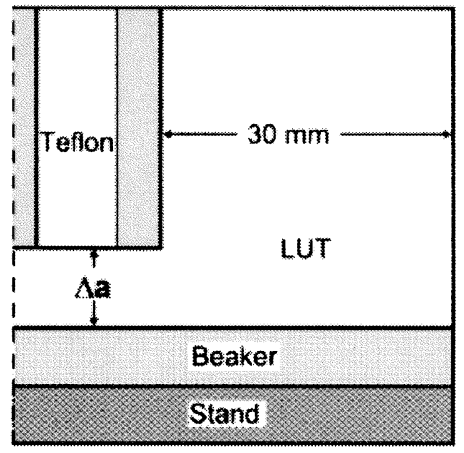

(b)

Fig. 2. FDTD models for investigating the influence of: (a) the radial boundary and (b) axial boundary. The dotted vertical line represents the center axis of the cylindrical coordinate system.

\section{METHODS}

In our experiments and numerical simulations, we use and model liquid tissue simulants rather than solid breast tissue specimens. Three standard liquids—ethanol, ${ }^{2}$ methanol, and

\footnotetext{
${ }^{2}$ Butanol is another commonly used reference liquid with low dielectric constant and conductivity. However, we found that the sensitivity of small-diameter coaxial probes immersed in butanol is too high at lower frequencies (particularly below $5 \mathrm{GHz}$ ) to yield reliable results.
} 


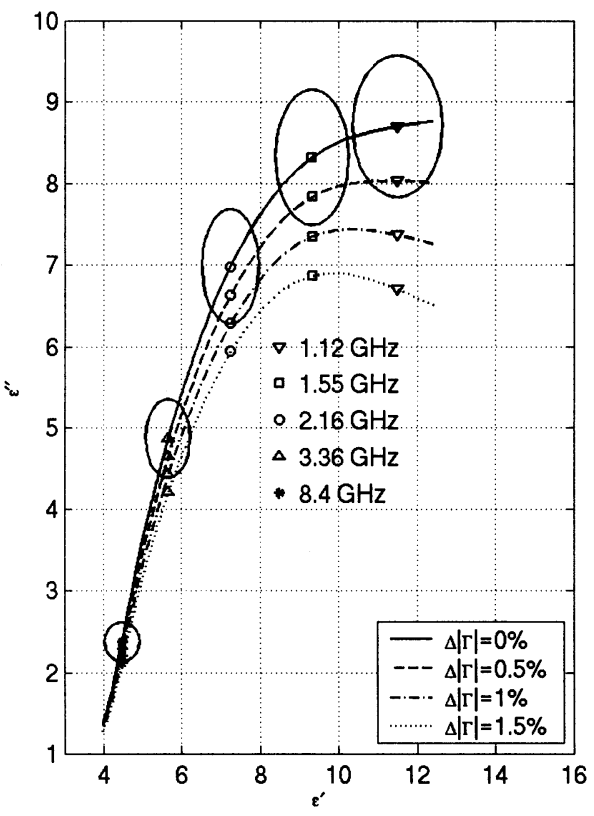

(a)

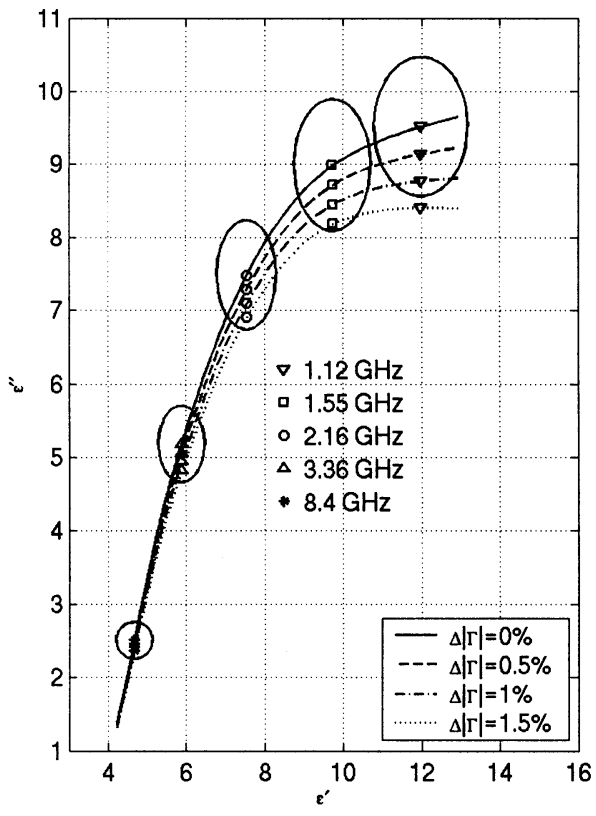

(c)

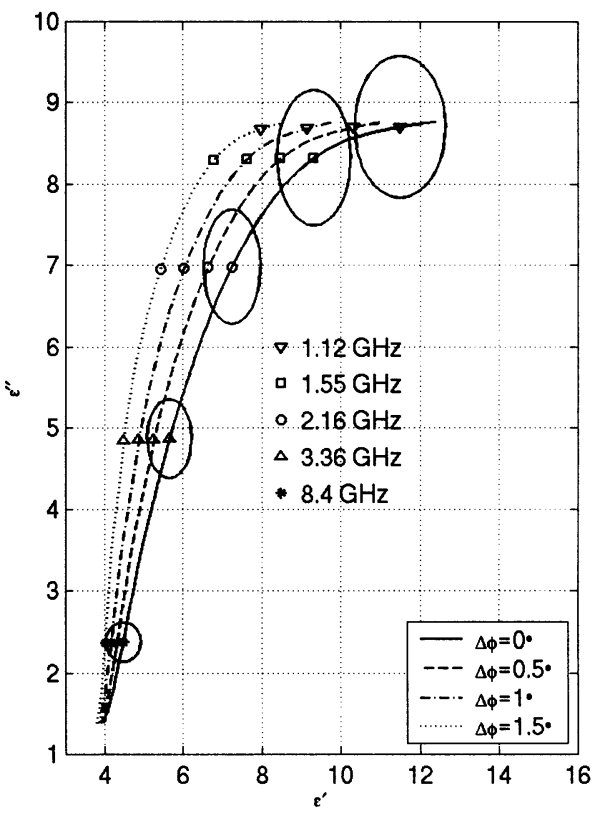

(b)

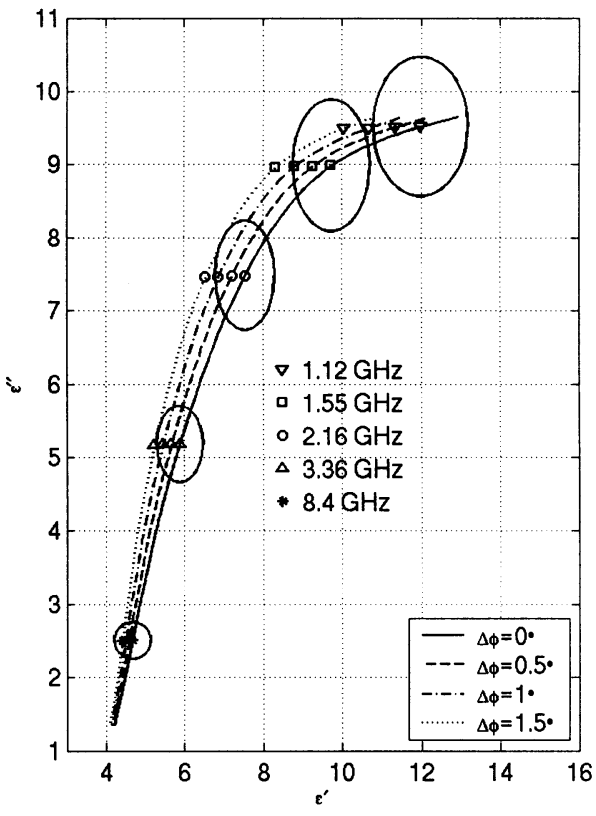

(d)

Fig. 3. Cole-Cole diagrams of complex permittivity as a function of frequency for three LUTs and two coaxial probe diameters. (a)-(d): ethanol. (a) and (b): $2.2 \mathrm{~mm}$. (c) and (d): $3.58 \mathrm{~mm}$. The black solid curve in each graph represents the permittivity obtained from the reference reflection coefficient. The dotted curves in each graph represent the permittivity obtained from incremental perturbations introduced in the magnitude or phase of the reference reflection coefficient. The ellipses centered at representative frequency points identify $\pm 10 \%$ error bounds on the complex permittivity.

deionized water-are selected to represent low, intermediate, and high values within the range of electrical properties expected for breast tissue. The dielectric properties of the glass beakers used to contain the liquid samples are similar to those of the trays on which breast tissue specimens are placed. Since it is difficult to position the probe exactly on the surface of the liquid samples, we decided to fully immerse the tip of the probes into the liquids, thereby removing all uncertainties involving the positioning of the probes. Admittedly, full immersion of the probe tip into a liquid bath is not precisely identical to pressing the probe tip into the soft surface of a breast tissue specimen. However, as shown in Section II-D, this slight difference in configuration has no significant effect on the determination of sensing volume guidelines. Thus, as configured, our setup is sufficiently realistic while offering a controlled test environment. It also permits us to move the probe with respect to the boundaries of a fixed-size sample, rather than change the actual size of the sample with respect to a fixed probe location. 


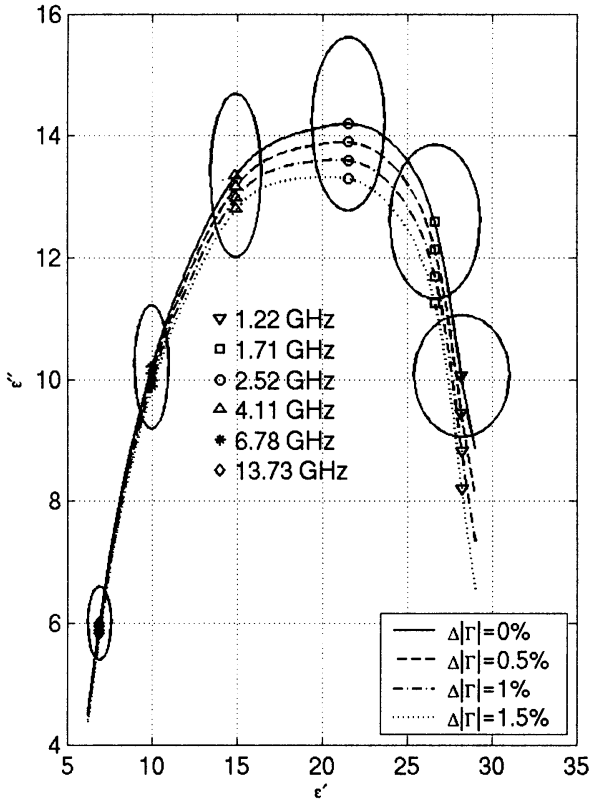

(e)

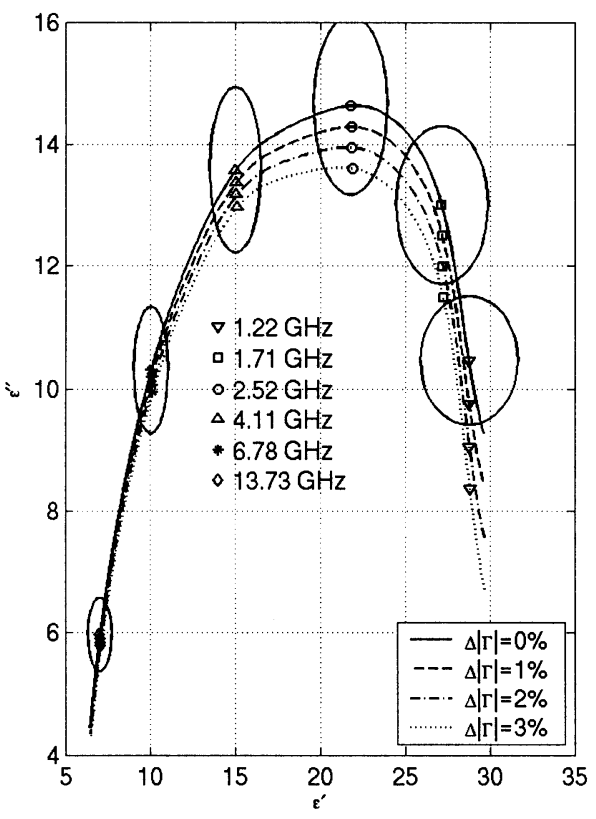

(g)

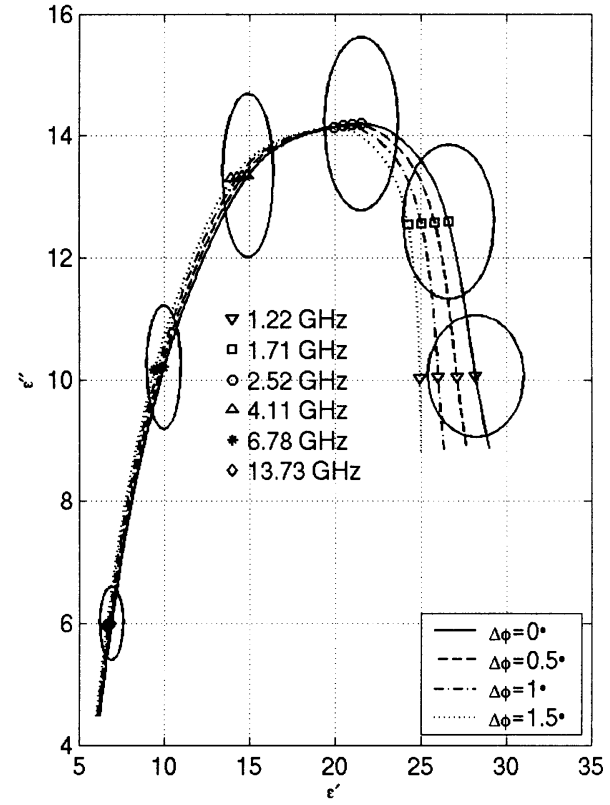

(f)

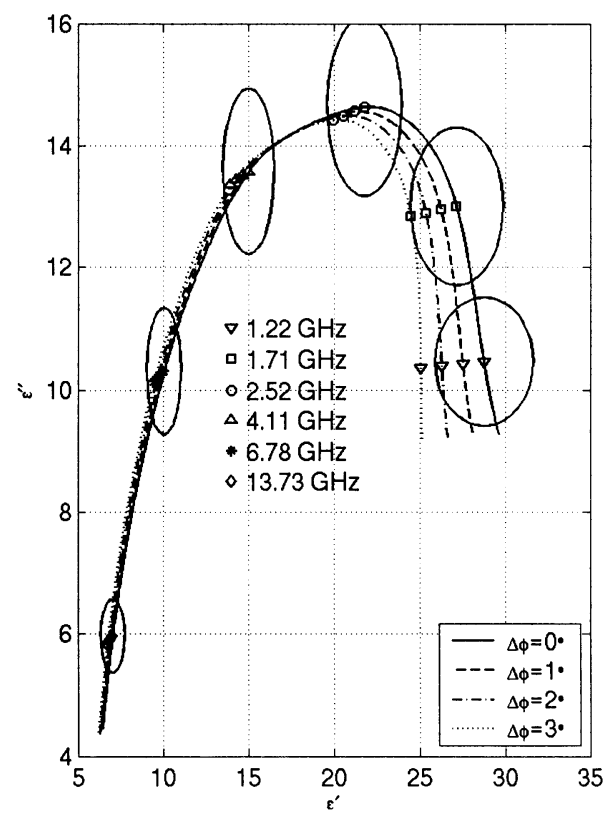

(h)

Fig. 3. (Continued). Cole-Cole diagrams of complex permittivity as a function of frequency for three LUTs and two coaxial probe diameters. (e)-(h): methanol. (e) and (f): $2.2 \mathrm{~mm}$. (g) and (h): $3.58 \mathrm{~mm}$. The black solid curve in each graph represents the permittivity obtained from the reference reflection coefficient. The dotted curves in each graph represent the permittivity obtained from incremental perturbations introduced in the magnitude or phase of the reference reflection coefficient. The ellipses centered at representative frequency points identify $\pm 10 \%$ error bounds on the complex permittivity.

We investigate the sensing volume of the probe by tracking changes in the reflection coefficient $\left(S_{11}\right.$ or $\left.\Gamma\right)$ as the fully immersed probe is placed at various distances from the edge or bottom of the beaker. In both experiments and numerical simulations, a reference reflection coefficient is obtained for each probe, liquid sample, and frequency by locating the probe sufficiently far from all sample boundaries in the axial (depth) and radial (transverse) directions. This reference reflection coefficient represents that which would be measured for a sample of infinite thickness and infinite transverse extent.
The probe is then positioned or modeled at the side or bottom boundary of the beaker and the reflection coefficient is measured or simulated at each location as the probe is moved in incremental steps away from the respective boundary. The details of the experiments and simulations are discussed further in Sections II-A and B, respectively. The resulting reflection coefficients are expressed as a percent error in magnitude and absolute error in phase with respect to the reference reflection coefficient. Plotted as a function of distance, this raw data illustrates the effect of the axial or radial bound- 


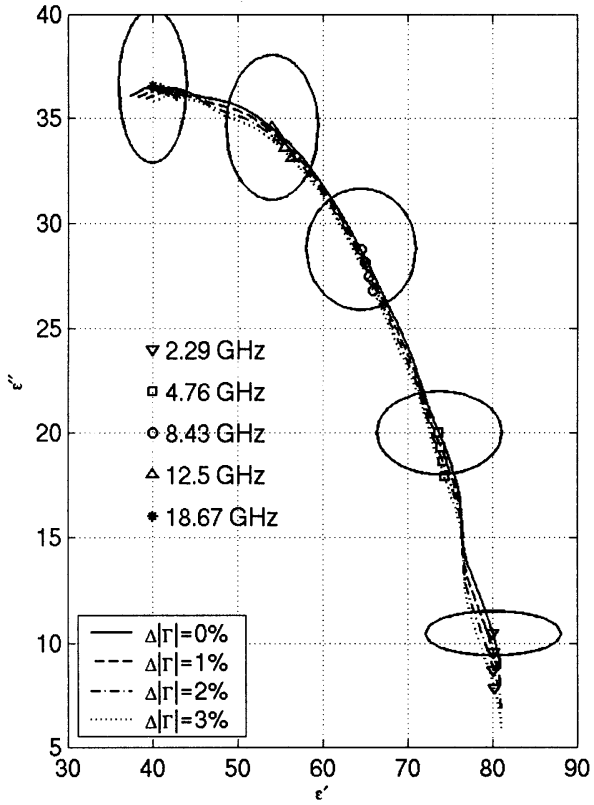

(i)

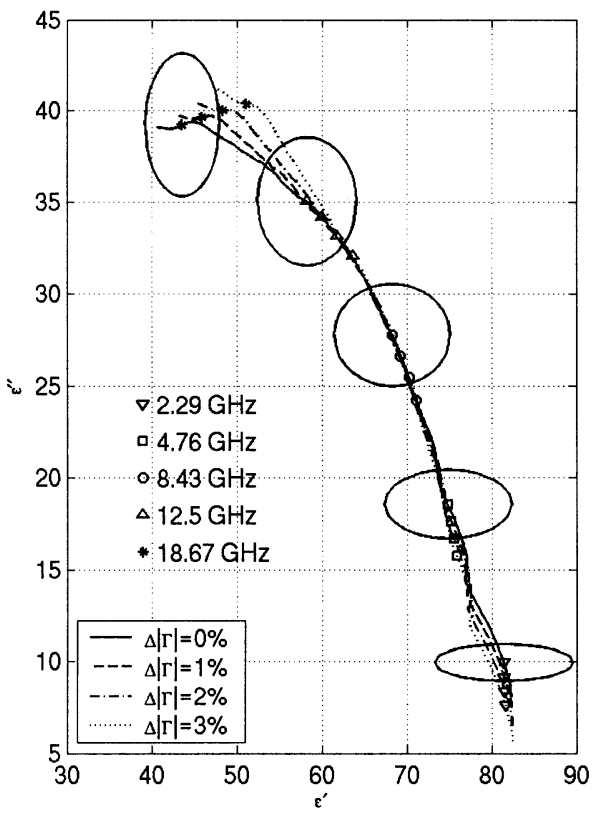

(k)

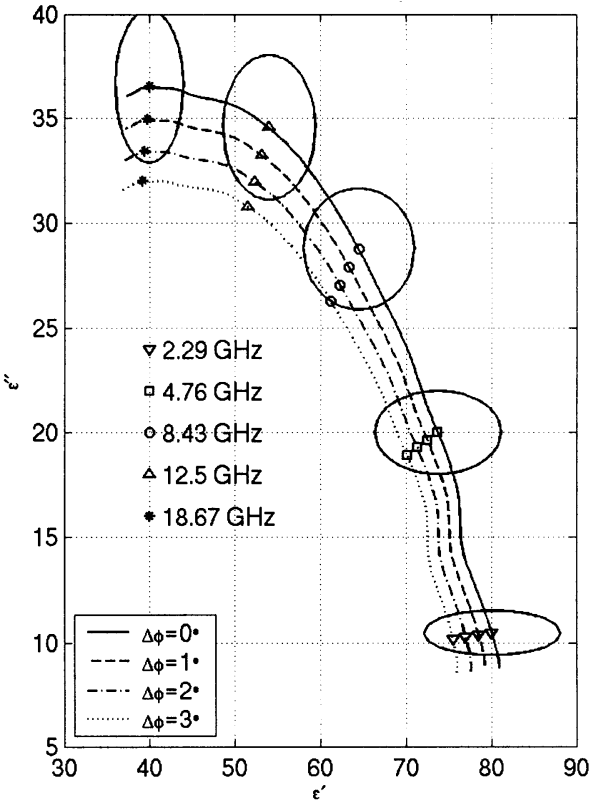

(j)

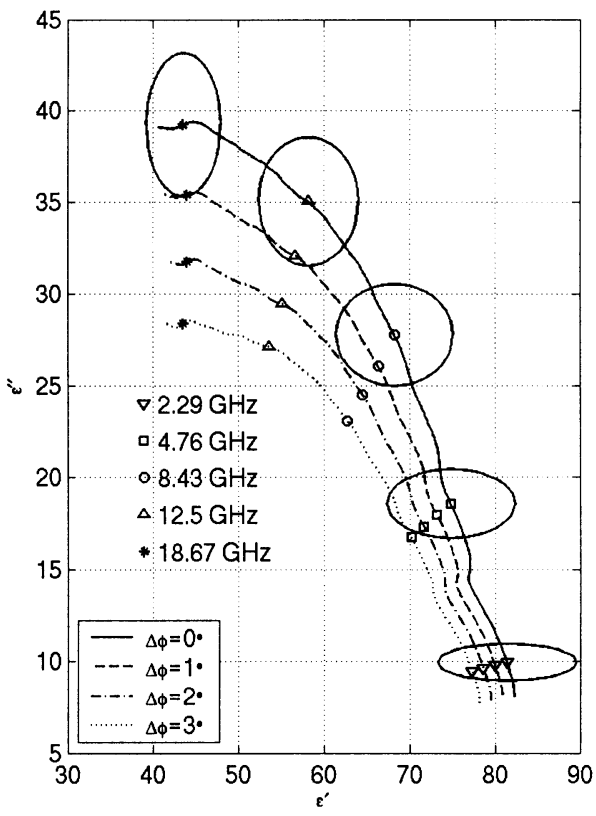

(1)

Fig. 3. (Continued). Cole-Cole diagrams of complex permittivity as a function of frequency for three LUTs and two coaxial probe diameters. (i)-(1): Deionized water. (i) and (j): $2.2 \mathrm{~mm}$. (k) and (l): $3.58 \mathrm{~mm}$. The black solid curve in each graph represents the permittivity obtained from the reference reflection coefficient. The dotted curves in each graph represent the permittivity obtained from incremental perturbations introduced in the magnitude or phase of the reference reflection coefficient. The ellipses centered at representative frequency points identify $\pm 10 \%$ error bounds on the complex permittivity.

TABLE I

$S_{11}$ Magnitude and Phase Error Thresholds That Limit the ERror on Real and Imaginary Parts of the Permittivity to $\pm 10 \%$

\begin{tabular}{c|c|c|c|c|c|c}
\hline & \multicolumn{2}{|c|}{$\mathrm{S}_{11}$ for Ethanol } & \multicolumn{2}{c|}{$\mathrm{S}_{11}$ for Methanol } & \multicolumn{2}{c}{$\mathrm{S}_{11}$ for Water } \\
\hline $\begin{array}{c}\text { Probe } \\
\text { Diameter }\end{array}$ & $\begin{array}{c}\text { Magnitude } \\
\text { error }( \pm \%)\end{array}$ & $\begin{array}{c}\text { Phase error } \\
( \pm \text { degrees })\end{array}$ & $\begin{array}{c}\text { Magnitude } \\
\text { error }( \pm \%)\end{array}$ & $\begin{array}{c}\text { Phase error } \\
( \pm \text { degrees })\end{array}$ & $\begin{array}{c}\text { Magnitude } \\
\text { error }( \pm \%)\end{array}$ & $\begin{array}{c}\text { Phase error } \\
( \pm \text { degrees })\end{array}$ \\
\hline $2.2 \mathrm{~mm}$ & $0.5-1.0$ & $\sim 0.5$ & $0.5-1.0$ & $1.0-1.5$ & $\sim 1.0$ & $2.0-3.0$ \\
\hline $3.58 \mathrm{~mm}$ & $1.0-1.5$ & $\sim 1.0$ & $\sim 1.5$ & $2.0-2.5$ & $\sim 1.0$ & $\sim 1.0$ \\
\hline
\end{tabular}

aries, regardless of the conversion method used to calculate permittivity. Finally, the reflection coefficient data is used to quantify the axial and radial extent of the sensing volume of a particular probe/sample configuration. As further explained in Section II-C, we identify the extent of the sensing volume as the smallest distance between the probe and boundary for which the magnitude and phase errors in the reflection coefficient remain below a defined error threshold. 


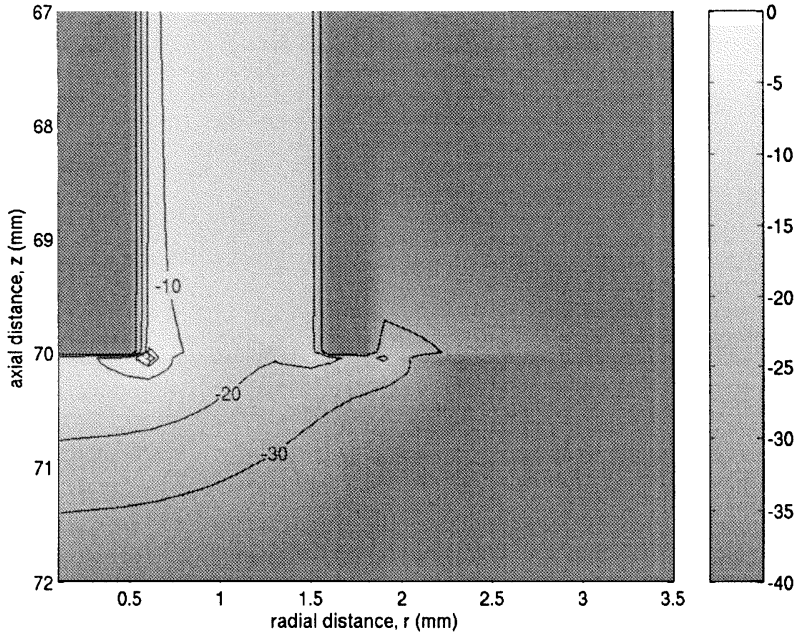

(a)

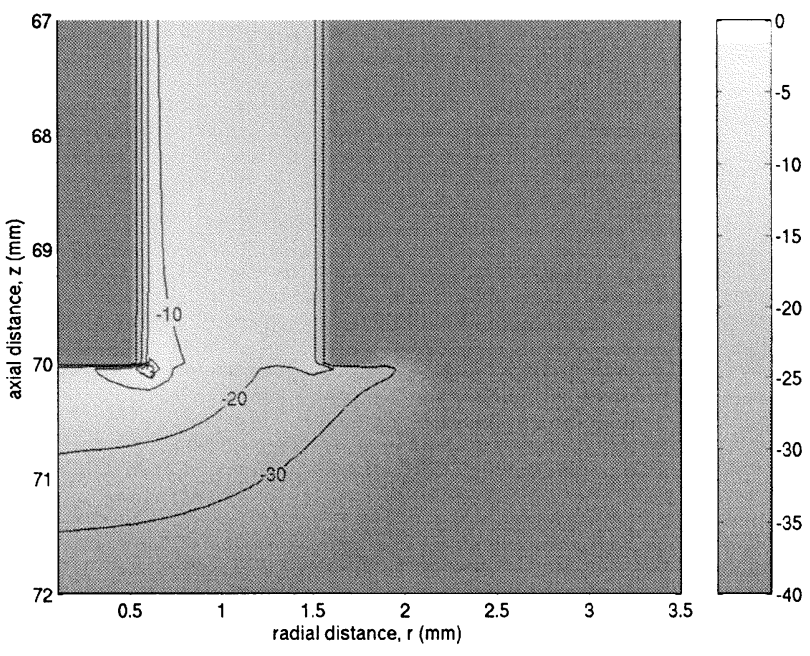

(b)

Fig. 4. Contour plots of the FDTD-computed electric field intensity (decibe scale) in a cross-sectional cut through the 3.58-mm-diameter probe. Here, the LUT is ethanol and the frequency of excitation is $2 \mathrm{GHz}$. (a) The probe is placed in contact with the surface of the LUT. (b) The probe is immersed at a depth of $10 \mathrm{~mm}$ in the LUT. The left boundary of each graph represents the center axis of the probe.

\section{A. Experimental Methods}

The experimental setup used at both UW and UC is shown in Fig. 1. The liquid under test (LUT) is held in a $600-\mathrm{mL}$ low-form glass beaker. A positioning stage is used to control the horizontal and vertical positions of the coaxial probe with submillimeter resolution in the LUT. The two coaxial probes used in this study are fabricated from commercial 2.2- and 3.58-mm-diameter semirigid copper/Teflon coaxial cables using subminiature A (SMA) connectors. A high-quality flexible cable is used to connect the probe to the VNA. An Agilent 8720D VNA is used at UW and an Agilent 8722D VNA is used at UC. All measurements are done at room temperature. The VNA is calibrated using a procedure similar to that described in [18].

The measurement sequence for determining the sensing volume properties of each probe is as follows. The probe is submerged in the beaker and air bubbles, which can introduce errors of up to $20 \%$ [23], are removed from the tip. The reference reflection coefficient is measured with the probe

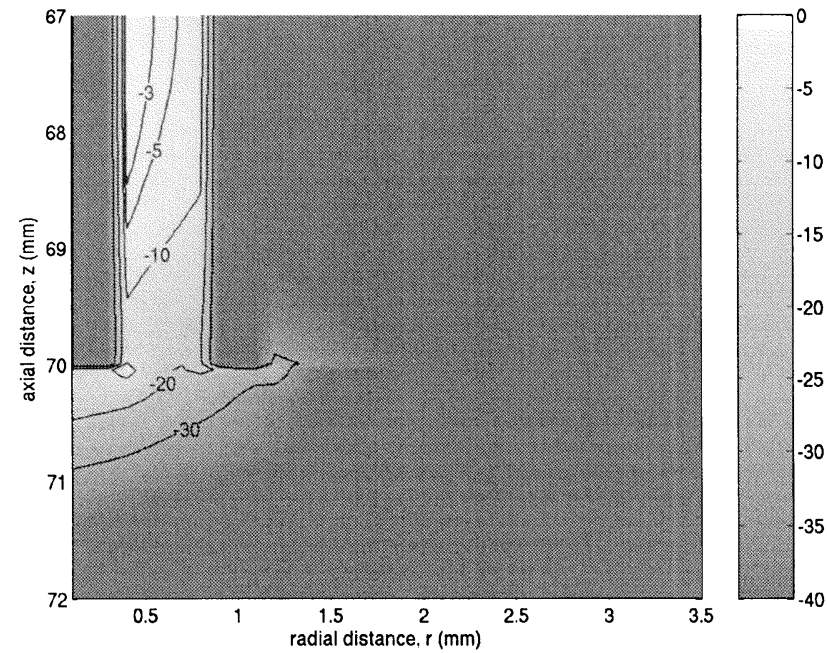

(a)

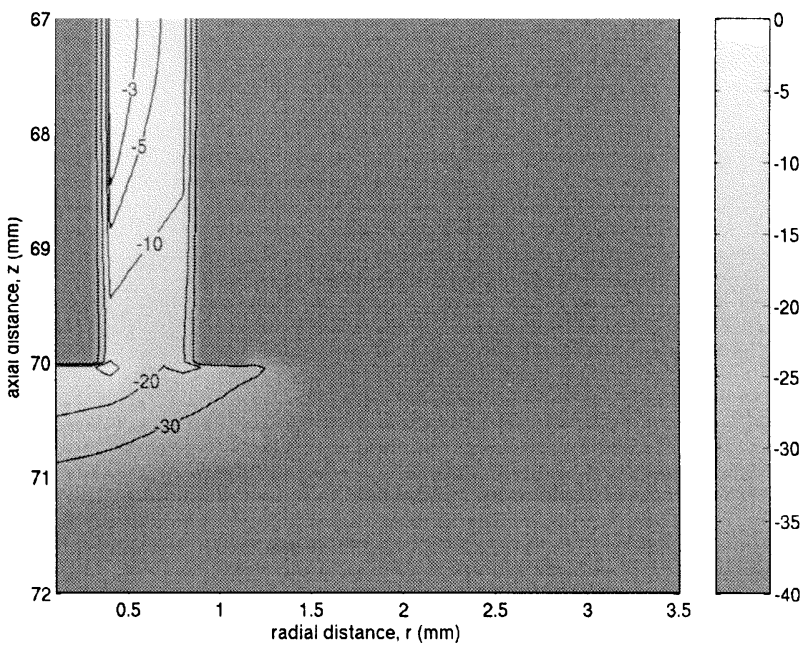

(b)

Fig. 5. Contour plots of the FDTD-computed electric field intensity (decibel scale) in a cross-sectional cut through the 2.2-mm-diameter probe. Here, the LUT is water and the frequency of excitation is $18 \mathrm{GHz}$. (a) The probe is placed in contact with the surface of the LUT. (b) The probe is immersed at a depth of $10 \mathrm{~mm}$ in the LUT.

positioned in the middle of the beaker where it is more than $30 \mathrm{~mm}$ from the sides and bottom of the beaker. To investigate the extent of the sensing volume in the radial direction, the reflection coefficient is measured with the probe positioned at incremental distances away from the side of the beaker, ranging from 0.0 to $10.0 \mathrm{~mm}$. To ensure that only the radial boundary effect is being measured, the tip of the probe is always more than $30 \mathrm{~mm}$ from the bottom of the beaker. To investigate the extent of the sensing volume in the axial direction, the reflection coefficient is measured with the probe positioned at incremental distances away from the bottom of the beaker, again, ranging from 0.0 to $10.0 \mathrm{~mm}$. To ensure that only the axial boundary effect is being measured, the probe is always more than $30 \mathrm{~mm}$ from the side of the beaker. For the axial case, the probe is moved in $0.5-\mathrm{mm}$ increments from the bottom boundary for the first $2.0 \mathrm{~mm}$, then $1.0-\mathrm{mm}$ increments for the remaining $8.0 \mathrm{~mm}$. For the radial case, the probe is moved in 1.0-mm increments from the side boundary. At each 


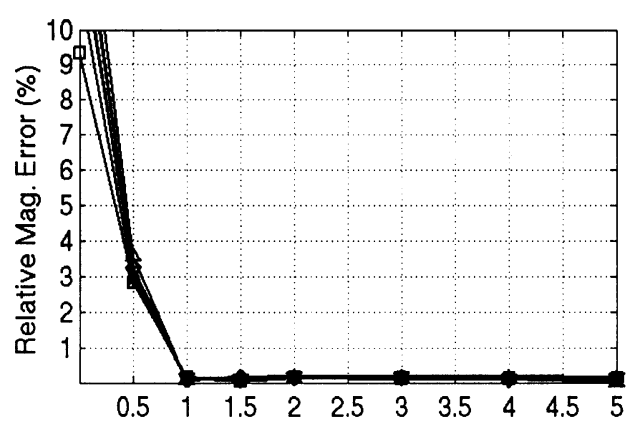

(a)

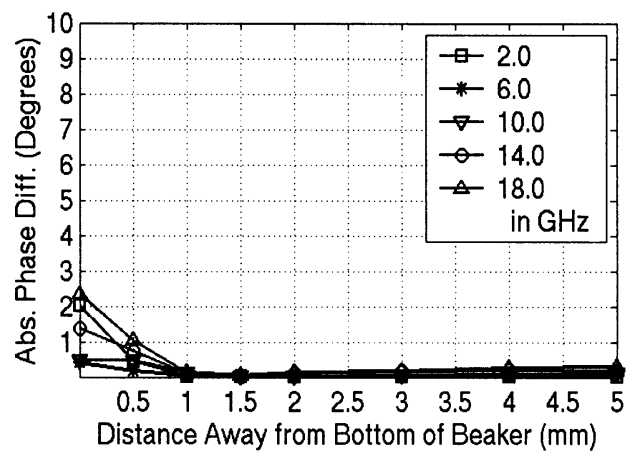

(b)

Fig. 6. Experimentally determined error in $S_{11}$ for ethanol as a function of distance between the end of the 2.2-mm-diameter probe and bottom of the beaker.

probe position, the reflection coefficient is recorded over the frequency range from 1 to $20 \mathrm{GHz}$.

\section{B. Simulation Methods}

Full-vector FDTD solutions of Maxwell's equations in cylindrical coordinates [24] are useful for studying open-ended coaxial probes, as demonstrated previously for the case of probes with finite-sized flanges [20], [25]. We assume that the geometry exhibits rotational symmetry, in which case, the azimuthal dependence of the vector field components can be expressed as a Fourier series and accounted for analytically. The FDTD models used at both universities are illustrated in Fig. 2. The dispersive properties of the liquids are incorporated using Debye parameters found in the literature (e.g., [26] gives $\varepsilon_{\infty}=4.2, \varepsilon_{s}=25.07, \tau=143.3 \mathrm{ps}$ for ethanol, $\varepsilon_{\infty}=5.7, \varepsilon_{s}=33.64, \tau=53 \mathrm{ps}$ for methanol, and $\varepsilon_{\infty}=5.2$, $\varepsilon_{s}=80.4$, and $\tau=9.45 \mathrm{ps}$ for water at $20^{\circ} \mathrm{C}$ ). The low-loss glass beaker and epoxy stand are modeled with frequency-independent dielectric properties (e.g., [27] gives $\varepsilon_{r}=4.82$ and $\sigma=0.01446(\mathrm{~S} / \mathrm{m})$ for Pyrex glass at $3 \mathrm{GHz}$, and [28] gives $\varepsilon_{r}=4.0$ and $\sigma=0.09444(\mathrm{~S} / \mathrm{m})$ for epoxy at $\left.5 \mathrm{GHz}\right)$. The thickness of the beaker sidewalls [see Fig. 2(a)] is $2.5 \mathrm{~mm}$ and the thickness of the beaker bottom [see Fig. 2(b)] is $3.0 \mathrm{~mm}$.

Simulations are conducted for probe positions similar to those used in the experiments. The reference reflection coefficient value is simulated for each LUT with the axial and radial boundaries $30 \mathrm{~mm}$ away. To study the sensing depth in the axial direction, we modify the distance $\Delta a$ between the tip of the probe and the axial boundary consisting of the bottom of the beaker and stand. To study the sensing volume in the radial direction, we modify the distance $\Delta r$ between the side of the probe and the

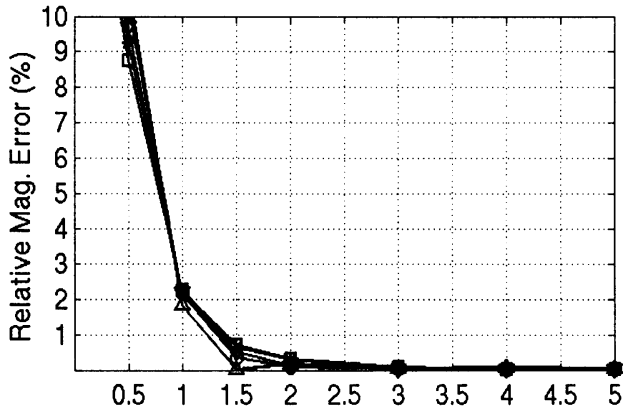

(a)

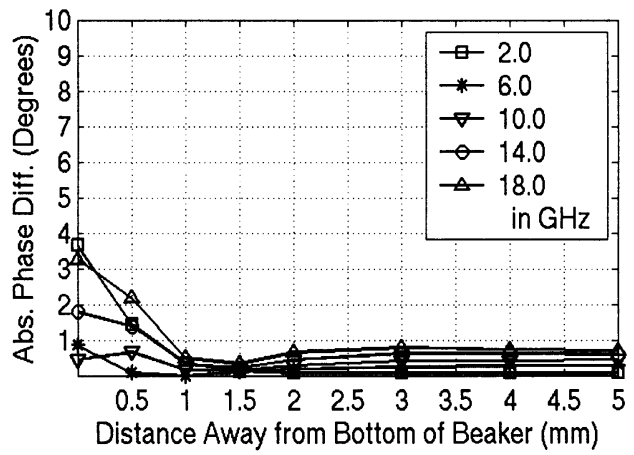

(b)

Fig. 7. Experimentally determined error in $S_{11}$ for ethanol as a function of distance between the end of the 3.58-mm-diameter probe and bottom of the beaker.

radial boundary, which consists of the beaker and air. It is important to note that, for small $\Delta r$, there is a significant difference between the simulation and experimental setup. In the simulation, the beaker and probe are concentric in the transverse plane due to the assumption of azimuthal symmetry. Conversely, in the experiment, the beaker and probe are highly nonconcentric due to the large difference in radii of curvature of the probe and beaker and the close proximity of the probe to the sidewall of the beaker. The simulated reflection coefficient will, therefore, exhibit a greater sensitivity to the presence of the boundary in the radial direction than the experimentally measured reflection coefficient.

\section{C. $S_{11}$ Error Threshold Definitions}

Once the percent errors in magnitude and absolute error in phase of the reflection coefficient are obtained as a function of probe position with respect to the LUT boundaries, the sensing volume of the probe is assessed. In this final step, we define the maximum acceptable level of error in the magnitude and phase of the reflection coefficient as the error threshold and identify the smallest distance between the probe and boundary for which the magnitude and phase errors in the reflection coefficient remain below this threshold. One approach to defining the error threshold is to make an arbitrary choice; e.g., one might define the threshold as a $1 \%$ error in magnitude and $1^{\circ}$ error in phase of the reflection coefficient. This approach is the simplest and can be directly applied to the graphs of reflection coefficient error as a function of distance presented in Section III. An alternative approach is to choose an acceptable level of error in the measured permittivity and work backward to determine the corresponding error threshold in the measured reflection coefficient. 


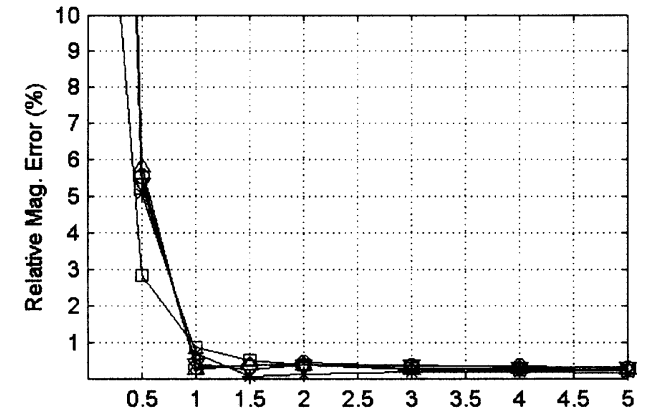

(a)

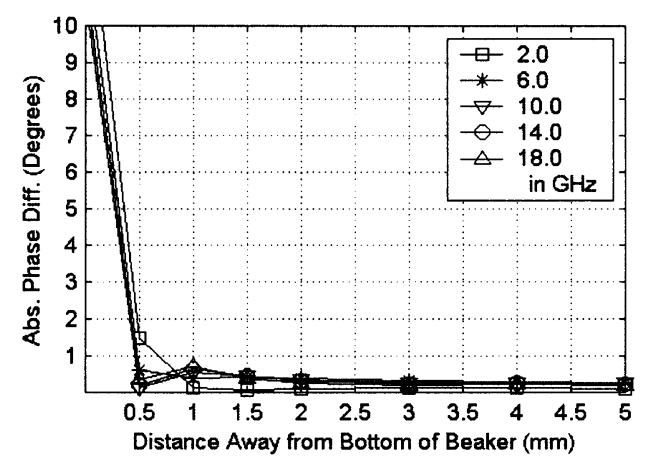

(b)

Fig. 8. Experimentally determined error in $S_{11}$ for methanol as a function of distance between the end of the 2.2-mm-diameter probe and bottom of the beaker.

This second approach is more meaningful, but less straightforward because it requires the use of a specific analytical or numerical method to relate the reflection coefficients to permittivity values.

We illustrate this second approach using one of the most accurate conversion methods - the rational function model (RFM) [18] - in conjunction with an innovative graphical technique based on Cole-Cole diagrams that permits straightforward determination of the error thresholds. Using the RFM, we convert the frequency-dependent reference reflection coefficient (that which is measured when the tip of the immersed probe is located in the center of the beaker, sufficiently far from all boundaries) into frequency-dependent complex permittivity reference data sets for each of the three liquids and each of the two probes. For each combination of liquid type and probe diameter, we artificially introduce incremental perturbations in the magnitude and phase of the reference reflection coefficient and convert the modified reflection coefficient to complex permittivity at each frequency sample across the $1-20-\mathrm{GHz}$ range.

In Fig. 3, the complex permittivity data sets for each liquid type and probe diameter are plotted in the form of Cole-Cole diagrams. The diagrams for ethanol, methanol, and deionized water are given in Fig. 3(a)-(d), (e)-(h), and (i)-(l), respectively. The black solid curve in each graph represents the complex permittivity obtained from the reference reflection coefficient data. Five ellipses are centered at representative frequency points along the locus of complex permittivity values. In this example, we have chosen $10 \%$ as the acceptable level of error in the real $\left(\varepsilon^{\prime}\right)$ and imaginary $\left(\varepsilon^{\prime \prime}\right)$ parts of the complex permittivity. Thus, the horizontal width of each ellipse represents a $\pm 10 \%$ variation in $\varepsilon^{\prime}$, while the vertical width of each ellipse

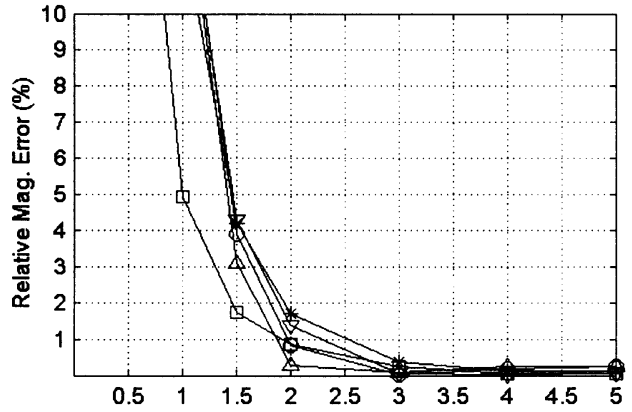

(a)

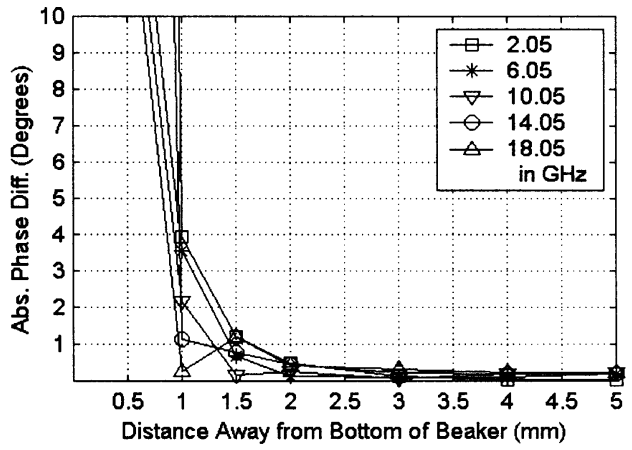

(b)

Fig. 9. Experimentally determined error in $S_{11}$ for methanol as a function of distance between the end of the 3.58-mm-diameter probe and bottom of the beaker.

represents a $\pm 10 \%$ variation in $\varepsilon^{\prime \prime}$. The ellipses enable us to graphically relate the acceptable level of error in permittivity to an upper bound on the error in the reflection coefficient. The various dotted curves in each diagram represent the complex permittivity that results from a positive incremental perturbation introduced in either the magnitude or phase of the reference reflection coefficient. Curves resulting from negative incremental perturbations would appear a similar distance away from the reference curve, but on the opposite side.

For example, consider Fig. 3(a), which graphs the complex permittivity loci for ethanol after a $0.0 \%,+0.5 \%,+1.0 \%$, and $+1.5 \%$ perturbation has been numerically introduced in the magnitude of the reference reflection coefficient measured by the 2.2-mm-diameter coaxial probe. At $1.12 \mathrm{GHz}$, only the first of the three dotted-line curves falls within the area of the ellipse. This indicates that a $+0.5 \%$ perturbation in the magnitude of the reflection coefficient yields less than a $10 \%$ error in the complex permittivity, but a $+1.0 \%$ perturbation does not. Similarly, a $-0.5 \%$ perturbation in the magnitude of the reflection coefficient would yield less than a $10 \%$ error, but a $-1.0 \%$ perturbation would not. Thus, the error threshold for the magnitude of $S_{11}$ falls between $\pm 0.5 \%$ and $\pm 1.0 \%$. Using this along with the remaining graphs in Fig. 3, we generate the data in Table I. By applying the threshold criteria of Table I to the graphs in Section III, we identify the sensing volume and the corresponding minimum specimen size required to achieve the desired accuracy.

We note that this error threshold analysis considers the contributions from the $S_{11}$ magnitude and phase errors separately. In other words, the thresholds in Table I represent the maximum allowed error in the magnitude assuming zero phase error, and 


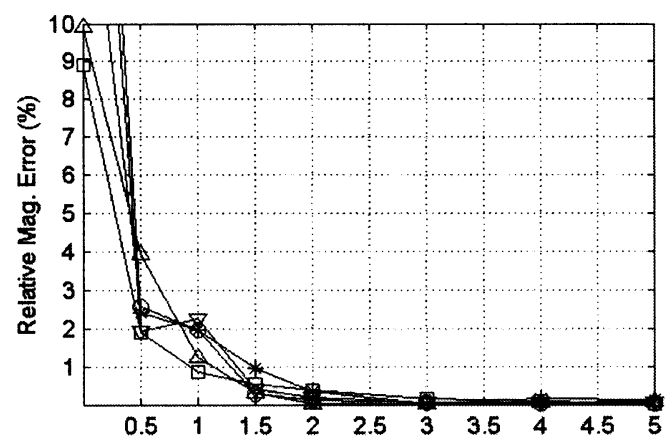

(a)

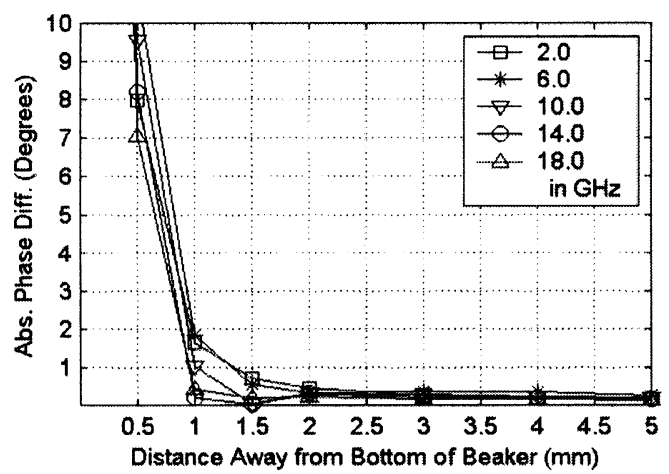

(c)

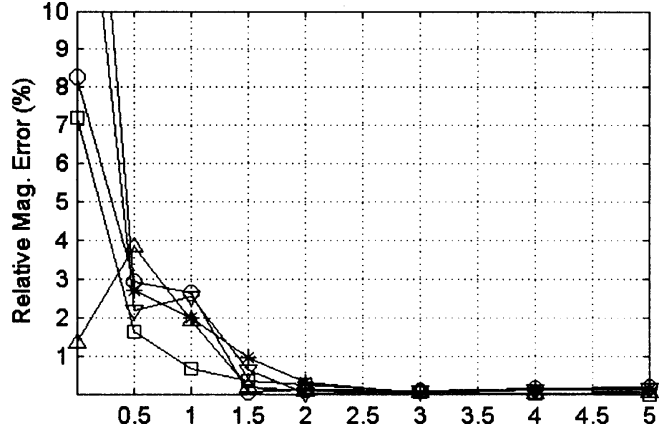

(b)

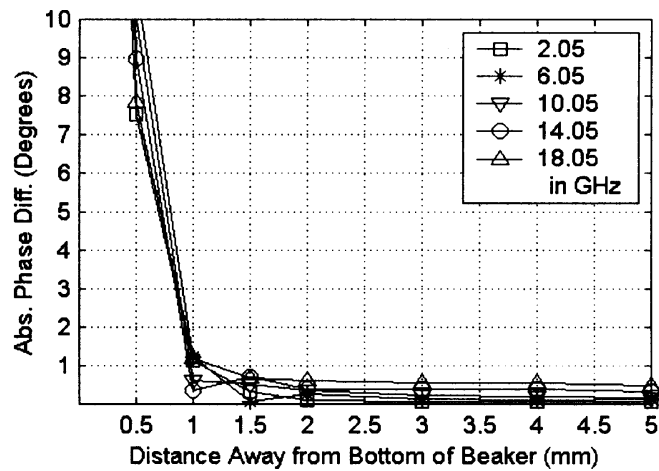

(d)

Fig. 10. Experimentally determined error in $S_{11}$ for deionized water as a function of distance between the end of the 2.2-mm-diameter probe and bottom of the beaker. Measurements were conducted at: (a) and (b) the UW and (c) and (d) UC.

vice versa. It does not consider the case where the effects of the errors in the magnitude and phase are combined. Consider a hypothetical measurement that introduces errors in the magnitude and phase of $S_{11}$ equal to the error thresholds listed in Table I for a specific probe/LUT pair. The combined effect of these errors would result in permittivity errors of $18 \%, 17 \%$, and $15 \%$ for ethanol, methanol, and water, respectively, for the $2.2-\mathrm{mm}$ probe. Similarly, for the $3.58-\mathrm{mm}$ probe, the permittivity errors would become $17 \%$ (ethanol), 15\% (methanol), and $13 \%$ (water) under such conditions. However, there is a practical problem with this approach to considering the combined effects of magnitude and phase. Our experimental results in Section III show that, in almost every instance, the magnitude error dominates the phase error. Further discussion of this point is reserved until the conclusion of Section III, where the analysis assumptions of Table I will be justified a posteriori.

\section{Validity of the Fully Immersed Probe Configuration}

For reasons explained at the beginning of this section, the tip of the coaxial probe is fully immersed in the LUT in the experimental setup and the corresponding FDTD simulations. This probe configuration differs slightly from the configuration encountered in practice where the probe tip is pressed against surface of a tissue specimen. To determine what impact, if any, this difference in configuration may have on the determination of the sensing volume, we have analyzed the distribution of the fringing fields beneath the probe using FDTD simulations. Here, we present two representative cases. Fig. 4 shows the magnitude of the electric field phasor at $2 \mathrm{GHz}$ with the $3.58-\mathrm{mm}$-diameter probe either placed on the surface [see Fig. 4(a)] or fully immersed [see Fig. 4(b)] in the liquid (ethanol). Fig. 5 shows similar results at $18 \mathrm{GHz}$ for the 2.2 -mm-diameter probe in water. To assess these differences, we consider the location of the $-30-\mathrm{dB}$ contour in the half-space beneath each probe. In the depth direction, there is less than a $0.1-\mathrm{mm}$ difference in the position of the contours. In the radial direction, there is only a $0.2-\mathrm{mm}$ difference for the larger probe in ethanol at $2 \mathrm{GHz}$ (Fig. 4) and a $0.1-\mathrm{mm}$ difference for the smaller probe in water at $18 \mathrm{GHz}$ (Fig. 5). These differences are insignificant for the purposes of quantifying the sensing volume. Hence, we conclude that the sensing volume of the probe is not affected by whether or not the probe is immersed in the LUT, and that our fully immersed probe configuration is valid for establishing sensing volume guidelines.

\section{RESULTS}

\section{A. Experimental}

The influence of the axial boundary on the reflection coefficient measurements is illustrated in Figs. 6-11. Fig. 10 shows excellent agreement between the UW and UC reflection-coefficient measurements for the 2.2-mm-diameter probe and deionized water as the LUT. We have seen this level of consistency in all of our data sets; therefore, aside from this example, we present graphs of reflection coefficient data from only one of the two measurement sites. A comparison of Figs. 6, 8, and 10 (or, likewise, a comparison of Figs. 7, 9, and 11) shows that, for a fixed probe size, the influence of the bottom of the beaker on $S_{11}$ increases as the dielectric-properties contrast between the LUT and beaker increases. A comparison between Figs. 6-11 shows that, for a fixed LUT, the influence of the bottom of the beaker on $S_{11}$ increases as the probe diameter increases. Figs. 6-11 


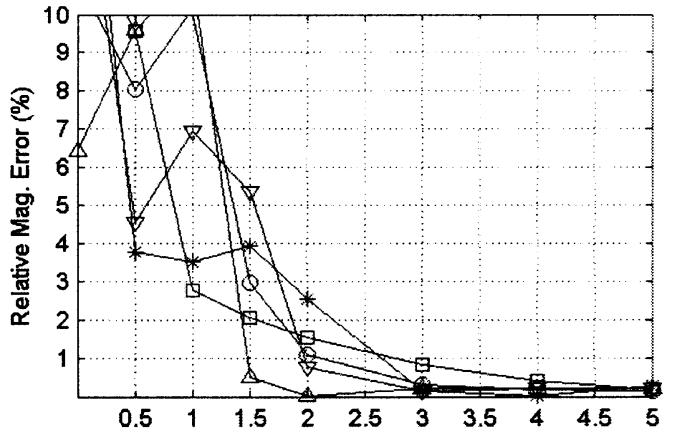

(a)

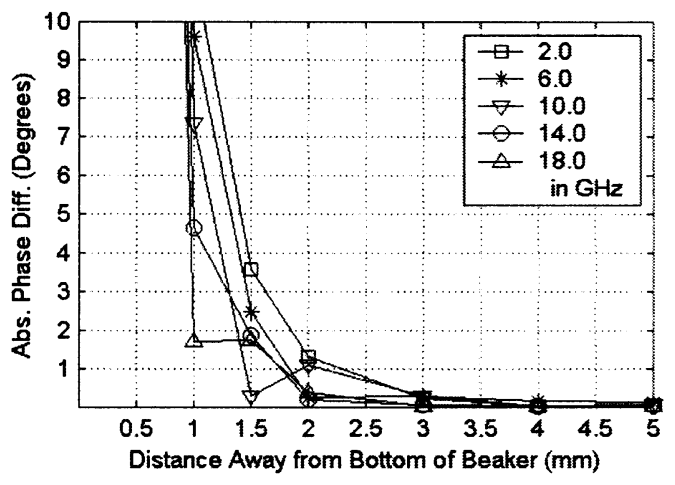

(b)

Fig. 11. Experimentally determined error in $S_{11}$ for deionized water as a function of distance between the end of the $3.58-\mathrm{mm}$-diameter probe and bottom of the beaker.

TABLE II

SENSING DePTh DETERMined From EXPERIMENTAL MEASUREMENTS AS A FUNCTION OF LUT AND PROBE APERTURE SIZE

\begin{tabular}{c|c|c|c}
\hline $\begin{array}{c}\text { Probe } \\
\text { diameter }\end{array}$ & Ethanol & Methanol & Water \\
\hline $2.2 \mathrm{~mm}$ & $0.75-1.0 \mathrm{~mm}$ & $1.0-1.5 \mathrm{~mm}$ & $\sim 1.5 \mathrm{~mm}$ \\
\hline $3.58 \mathrm{~mm}$ & $1.25-1.5 \mathrm{~mm}$ & $\sim 2.25 \mathrm{~mm}$ & $\sim 2.5-3.0 \mathrm{~mm}$ \\
\hline
\end{tabular}

indicate that the influence of the axial boundary does not vary significantly over the frequency range of interest.

Table II summarizes the estimates for the sensing depth of the two probes. To illustrate how the reflection-coefficient error thresholds defined in Table I are applied to the data of Figs. 6-11 to determine the sensing depth, we consider the example of Fig. 9 for methanol and the 3.58-mm-diameter probe. Fig. 9(a) suggests that a minimum distance of approximately $2.25 \mathrm{~mm}$ is required to ensure that the error in the magnitude of the reflection coefficient is below the threshold of $1.5 \%$ (from Table I) over the 1-20-GHz frequency range. Fig. 9(b) suggests that a minimum distance of approximately $1.25 \mathrm{~mm}$ is required to ensure that the phase error remains below the threshold of $2.0^{\circ}-2.5^{\circ}$ (from Table I). Therefore, the sensing depth is identified as $2.25 \mathrm{~mm}$ - the larger of the two distances. We note that the error threshold for the magnitude determines the sensing depth in this example. According to the data of Table II, the sensing depth is greater for the larger diameter probe. The sensing depth is clearly a function of the LUT and is greatest for the case of water, which has the highest dielectric properties of the three LUTs, as well as the largest dielectric-properties contrast with the adjacent medium (the beaker).

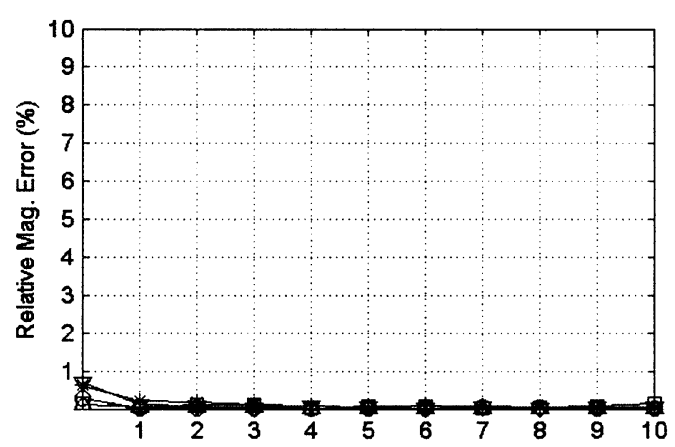

(a)

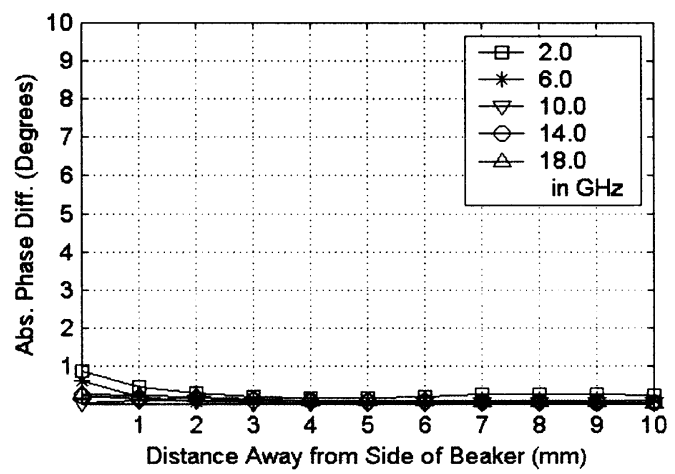

(b)

Fig. 12. Experimentally determined error in $S_{11}$ for deionized water as a function of distance between the outer conductor of the 3.58-mm-diameter probe and the side of the beaker.

The influence of the radial boundary on the measured reflection coefficient is illustrated in Fig. 12 for deionized water (the LUT that exhibits the largest dielectric-properties contrast with the beaker). We note that this is the case of a nonconcentric radial boundary, i.e., a large-diameter circular boundary that is not concentric with the small-diameter probe in the transverse plane. The influence is minimal, even when the probe is in direct contact with the side of the beaker. For all liquids and probes, there is no required distance of separation between the outer conductor of the probe and nonconcentric radial boundary. This suggests that accurate measurements can be made with the probe located at the edge of a specimen or region of homogeneous tissue as long as the radius of curvature of the boundary is much larger than that of the probe's outer conductor.

\section{B. Simulation}

Fig. 13 illustrates the influence of the axial boundary on the reflection coefficient, as characterized numerically via FDTD simulations. A comparison between Figs. 10 and 13, both of which report data for the 2.2-mm-diameter probe in deionized water, illustrates the excellent agreement we have obtained between our experimental and FDTD results for the sensing depth of the probes. We have also made a direct comparison of the measured and simulated reflection coefficients used as the reference data in creating the error plots of Figs. 10 and 13. Overall, the agreement between measured and simulated reference reflection coefficients is within $2 \%$ for the magnitude and $5^{\circ}$ for the phase. Estimates of the sensing depth of the two probes determined from the simulation data are summarized in Table III. 


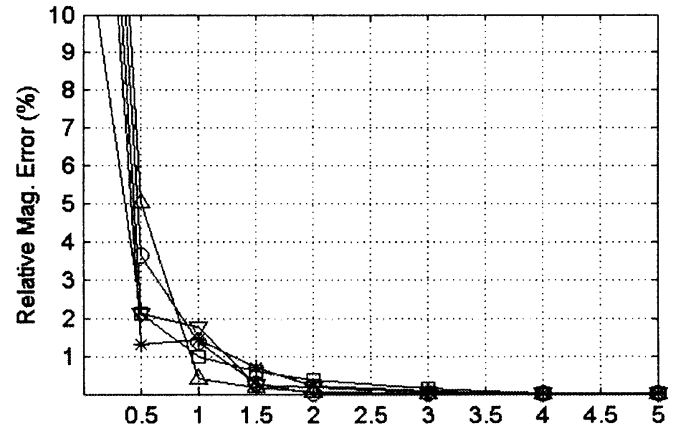

(a)

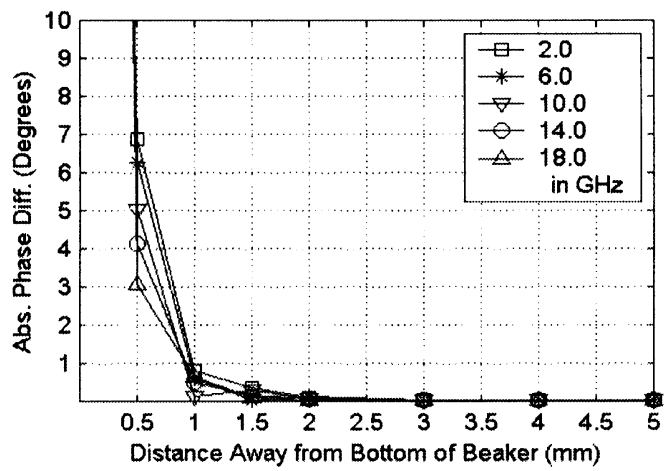

(b)

Fig. 13. FDTD-computed error in $S_{11}$ for deionized water as a function of distance between the end of the 2.2-mm-diameter probe and the bottom of the beaker.

TABLE III

SENSING DEPTh DETERMined From FDTD Simulations as A FUNCTION OF LUT AND PROBE APERTURE SIZE

\begin{tabular}{c|c|c|c}
\hline $\begin{array}{c}\text { Probe } \\
\text { diameter }\end{array}$ & Ethanol & Methanol & Water \\
\hline $2.2 \mathrm{~mm}$ & $0.5-1.0 \mathrm{~mm}$ & $1.0-1.5 \mathrm{~mm}$ & $1.25-1.5 \mathrm{~mm}$ \\
\hline $3.58 \mathrm{~mm}$ & $1.0-1.5 \mathrm{~mm}$ & $\sim 1.75 \mathrm{~mm}$ & $\sim 2.5 \mathrm{~mm}$ \\
\hline
\end{tabular}

Excellent agreement is noted between Table II (experimental results) and Table III (simulation results).

The small differences between experimental and simulation results may be attributed to two potential sources of discrepancy. First, the probes are not flawless, but they are represented as such in the simulation. Imperfections in the probes can introduce variations in the experimental results [29]. Secondly, the material properties assumed in the simulations may not exactly represent the actual material properties. For example, the beaker and stand material parameters are estimated from data in the literature for similar materials. Also, the dispersive properties of the liquids are incorporated in the simulations using a single-pole Debye model, whereas their behavior, particularly water, may be better represented with a Cole-Davidson dispersion model.

The influence of the concentric radial boundary on the simulated reflection coefficient is illustrated in Fig. 14. As expected, this influence is much stronger than that of the nonconcentric radial boundary. Estimates of each probe's radial sensing dimension ( $\Delta r$, as labeled in Fig. 2$)$ are summarized in Table IV. The radial sensing dimension is greatest for the larger diameter probe and the LUT with the highest dielectric properties and

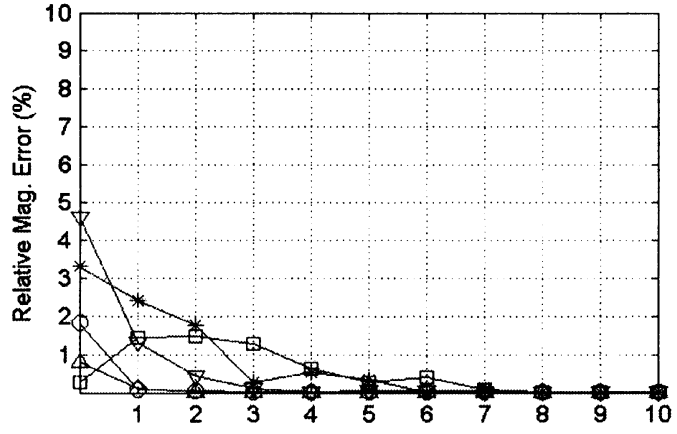

(a)

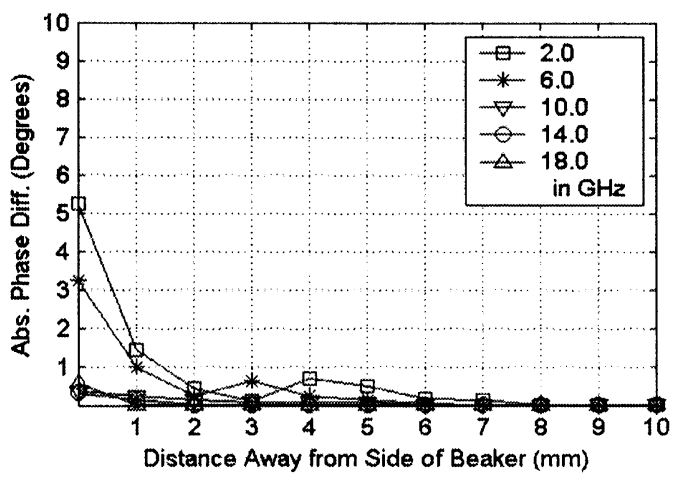

(b)

Fig. 14. FDTD-computed error in $S_{11}$ for deionized water as a function of distance between the outer conductor of the 3.58-mm-diameter probe and side of the beaker.

TABLE IV

RADIAL SENSING DimENSION DETERMINED FROM FDTD SiMULATIONS AS A FUNCTION OF LUT AND PROBE APERTURE SIZE

\begin{tabular}{c|c|c|c}
\hline $\begin{array}{c}\text { Probe } \\
\text { diameter }\end{array}$ & Ethanol & Methanol & Water \\
\hline $2.2 \mathrm{~mm}$ & $<0.5 \mathrm{~mm}$ & $0.5-1.0 \mathrm{~mm}$ & $\sim 1.25 \mathrm{~mm}$ \\
\hline $3.58 \mathrm{~mm}$ & $<0.5 \mathrm{~mm}$ & $\sim 1.0 \mathrm{~mm}$ & $3.5-3.75 \mathrm{~mm}$ \\
\hline
\end{tabular}

the largest dielectric-properties contrast with the surrounding medium (the beaker). As explained in Section II, our FDTD simulations cannot be directly compared with our experimental measurements for this case because of the two different ways in which the beaker encircles the probe in the transverse plane.

Finally, we return to the discussion brought up at the conclusion of Section II-C regarding the validity of ignoring the combined effects of $S_{11}$ magnitude and phase errors in determining error thresholds. In both the UW and UC data sets, thresholds for the magnitude error dominate the phase error in determining the sensing dimensions in almost every instance. The exception is seen in Fig. 6 for the case of the 2.2-mm probe with ethanol as the LUT, where the magnitude and phase error thresholds are reached at approximately the same distance away $(0.75-1.0 \mathrm{~mm})$ from the bottom of the beaker. Fig. 6 also shows, however, that both the magnitude and phase errors drop to negligible values for distances greater than $1.0 \mathrm{~mm}$. This latter point is particularly relevant in light of the sensing depths identified for the other two LUTs since they override the ethanol results in establishing guidelines for the minimum specimen thickness, as discussed in Section IV. Hence, the analysis assumptions of Table I are justified a posteriori. 


\section{CONCLUSIONS}

Consistency in the results obtained experimentally and through simulations, both of which were conducted independently at two different universities, gives us a high level of confidence in the reliability of the data that has been reported here. From Tables II and III, we conclude that, for frequencies between 1-20 GHz, the thickness of the breast tissue specimen (or extent of homogeneity in the case of a larger specimen) should be greater than $3.0 \mathrm{~mm}$ for a $3.58-\mathrm{mm}$ probe and greater than $1.5 \mathrm{~mm}$ for a $2.2-\mathrm{mm}$ probe in order to bound the permittivity errors due to the finite size of the specimen to approximately $10 \%$. When the size of the specimen (or the size of the homogeneous region of tissue) is such that its margins are concentric with the probe in the transverse plane, the $3.58-\mathrm{mm}$-diameter probe should be at least $\Delta r=3.75 \mathrm{~mm}$ from all edges and the 2.2-mm-diameter probe should be at least $\Delta r=1.25 \mathrm{~mm}$ away from all edges. Thus, the full transverse extent of the specimen (or homogeneous region of tissue) should be at least $1.1 \mathrm{~cm}$ for the $3.58-\mathrm{mm}$ probe and at least $5 \mathrm{~mm}$ for the $2.2-\mathrm{mm}$ probe in order to bound the permittivity error to approximately $10 \%$. For much larger specimens (or larger regions of homogeneous tissue), accurate measurements may be achieved regardless of how close the outer diameter of the probe is to the margin.

\section{ACKNOWLEDGMENT}

The authors would like to thank R. Hagl, AGFA-Gevaert AG, Munich, Germany, for his technical support in processing the large number of data sets involved in this investigation.

\section{REFERENCES}

[1] Inst. Med., Mammography and Beyond: Developing Technologies for the Early Detection of Breast Cancer. Washington, DC: Nat. Academy Press, 2000

[2] S. C. Hagness, A. Taflove, and J. E. Bridges, "Two-dimensional FDTD analysis of a pulsed microwave confocal system for breast cancer detection: Fixed-focus and antenna-array sensors," IEEE Trans. Biomed. Eng., vol. 45, pp. 1470-1479, Dec. 1998.

[3] X. Li and S. C. Hagness, "A confocal microwave imaging algorithm for breast cancer detection," IEEE Microwave Wireless Comp. Lett., vol. 11, pp. 130-132, Mar. 2001.

[4] P. M. Meaney, M. W. Fanning, D. Li, S. P. Poplack, and K. D. Paulsen, "A clinical prototype for active microwave imaging of the breast," IEEE Trans. Microwave Theory Tech., vol. 48, pp. 1841-1853, Nov. 2000.

[5] E. C. Fear and M. A. Stuchly, "Microwave detection of breast cancer," IEEE Trans. Microwave Theory Tech., vol. 48, pp. 1854-1863, Nov. 2000.

[6] G. Ku and L. H. V. Wang, "Scanning microwave-induced thermoacoustic tomography: Signal, resolution, and contrast," Med. Phys., vol. 28, pp. 4-10, Jan. 2001.

[7] R. A. Kruger, K. D. Miller, H. E. Reynolds, W. L. Kiser, D. R. Reinecke, and G. A. Kruger, "Breast cancer in vivo: Contrast enhancement with thermoacoustic CT at $434 \mathrm{MHz}$-feasibility study," Radiology, vol. 216, pp. 279-283, 2000.

[8] A. J. Fenn, G. L. Wolf, and R. M. Fogle, "An adaptive microwave phased array for targeted heating of deep tumours in intact breast: Animal study results," Int. J. Hyperthermia, vol. 15, no. 1, pp. 45-61, 1999.

[9] S. S. Chaudhary, R. K. Mishra, A. Swarup, and J. M. Thomas, "Dielectric properties of normal and malignant human breast tissues at radiowave and microwave frequencies," Indian J. Biochem. Biophys., vol. 21, pp. 76-79, Feb. 1984.

[10] A. J. Surowiec, S. S. Stuchly, J. R. Barr, and A. Swarup, "Dielectric properties of breast carcinoma and the surrounding tissues," IEEE Trans. Biomed. Eng., vol. 35, pp. 257-263, Apr. 1988.
[11] A. M. Campbell and D. V. Land, "Dielectric properties of female human breast tissue measured in vitro at $3.2 \mathrm{GHz}$," Phys. Med. Biol., vol. 37, no. 1, pp. 193-210, 1992.

[12] W. T. Joines, Y. Z. Dhenxing, and R. L. Jirtle, "The measured electrical properties of normal and malignant human tissues from 50 to $900 \mathrm{MHz}$," Med. Phys., vol. 21, pp. 547-550, Apr. 1994.

[13] M. A. Stuchly and S. S. Stuchly, "Coaxial line reflection methods for measuring dielectric properties of biological substances at radio and microwave frequencies-A review," IEEE Trans. Instrum. Meas., vol. IM-29, pp. 176-183, Sept. 1980.

[14] T. W. Athey, M. A. Stuchly, and S. S. Stuchly, "Measurement of radio frequency permittivity of biological tissues with an open-ended coaxial line: Part I," IEEE Trans. Microwave Theory Tech., vol. MTT-30, pp. $82-87,1982$.

[15] D. Misra, "A quasistatic analysis of open-ended coaxial line," IEEE Trans. Microwave Theory Tech., vol. MTT-35, pp. 925-928, Oct. 1987.

[16] F. M. Ghannouchi and R. G. Bosisio, "Measurements of microwave permittivity using a six-port reflectometer with open-ended coaxial line," IEEE Trans. Instrum. Meas., vol. 38, pp. 505-508, June 1989.

[17] C. Gabriel, T. Y. A. Chan, and E. H. Grant, "Admittance models for open ended coaxial probes and their place in dielectric spectroscopy," Phys Med. Biol, vol. 39, pp. 2183-2200, 1994.

[18] J. M. Anderson, C. L. Sibbald, and S. S. Stuchly, "Dielectric measurements using a rational function model," IEEE Trans. Microwave Theory Tech., vol. 42, pp. 199-204, Feb. 1994.

[19] G. G. Stuchly, "Numerical analysis of open-ended coaxial lines," IEEE Trans. Microwave Theory Tech., vol. MTT-31, pp. 380-384, May 1983.

[20] P. DeLanghe, L. Martens, and D. De Zutter, "Design rules for an experimental setup using an open-ended coaxial probe based on theoretical modeling," IEEE Trans. Instrum. Meas., vol. 43, pp. 810-817, Dec. 1994.

[21] M. A. El-Rayes and F. T. Ulaby, "Microwave dielectric spectrum of vegetation-Part I: Experimental observations," IEEE Trans. Geosci. Remote Sensing, vol. GE-25, pp. 541-549, Sept. 1987.

[22] S. Hoshina, Y. Kanai, and M. Miyakawa, "A numerical study on the measurement region of an open-ended coaxial probe used for complex permittivity measurement," IEEE Trans. Magn., vol. 37, pp. 3311-3314, Sept. 2001.

[23] P. De Langhe, K. Blomme, L. Martens, and D. De Zutter, "Measurement of low-permittivity materials based on a spectral-domain analysis for the open-ended coaxial probe," IEEE Trans. Instrum. Meas., vol. 42, pp. 879-886, Oct. 1993.

[24] A. Taflove and S. Hagness, Computational Electrodynamics: The FiniteDifference Time-Domain Method, 2nd ed. Boston, MA: Artech House, 2000.

[25] M. Okoniewski, J. M. Anderson, E. Okoniewska, K. Caputa, and S. S. Stuchly, "Further analysis of open-ended dielectric sensors," IEEE Trans. Microwave Theory Tech., vol. 43, pp. 1986-1989, Aug. 1995.

[26] "Tables of dielectric dispersion data for pure liquids and dilute solutions," NIST, Boulder, CO, Circular 589, 1958.

[27] D. M. Pozar, Microwave Engineering, 2nd ed. New York: Wiley, 1998.

[28] J. Baker-Jarvis, B. Riddle, and M. D. Janezic, "Dielectric and magnetic properties of printed wiring boards and other substrate materials," RF Tech. Div., U.S. Dept. Commerce, Boulder, CO, NIST Tech. Note 1512, 1999.

[29] D. Popovic and M. Okoniewski, "Effects of mechanical flaws in open-ended coaxial probes for dielectric spectroscopy," IEEE Microwave Wireless Comp. Lett., vol. 12, pp. 401-403, Oct. 2002.

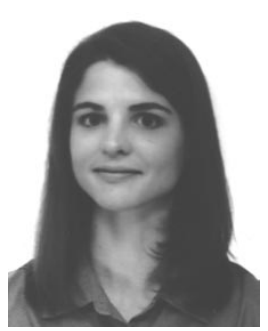

Dina M. Hagl (S'00) received the B.S. and M.S. degrees in electrical engineering from the University of Wisconsin-Madison, in 2000 and 2001, respectively.

She is currently a Scientific Investigator with the Aerospace and Astronautics Department, Bundeswehr-Universitaet, Munich, Germany where she is engaged in microwave engineering design for European Space Agency missions including Rosetta, Mars Express, and Venus Express.

Ms. Hagl is a member of Tau Beta Pi and Eta Kappa Nu. She has served in numerous leadership roles in the Wisconsin Alpha Chapter of Tau Beta Pi and the UW-Madison Student Chapter of the Society of Women Engineers. She was the recipient of a 2000 University of Wisconsin Hilldale Award for Undergraduate Research. 


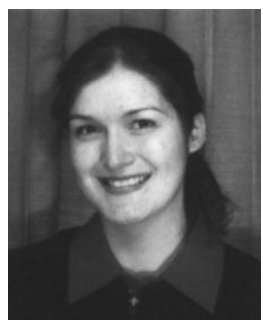

Dijana Popovic (S'00) received the B.S. and M.S. degrees in electrical engineering from the University of Calgary, Calgary, AB, Canada, in 2000 and 2002 , respectively, and is currently working toward the Ph.D. degree in electrical engineering at the University of Calgary.

Her research interests are medical applications of microwave technologies, particularly for microwave breast cancer detection.

Ms. Popovic is on the Executive Committees of the IEEE Engineering in Medicine and Biology Society (EMBS) Student Club and the Engineering Graduate Student's Association at the University of Calgary. She was the recipient of the 2002 IEEE Microwave Theory and Techniques Society (IEEE MTT-S) Graduate Scholarship, the National Sciences and Engineering Research Council (NSERC) Award, and the Alberta Informatics Circle of Research Excellence (iCORE) Scholarship.

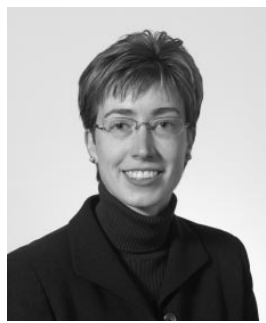

Susan C. Hagness (S'91-M'98) received the B.S. degree (with highest honors) and the Ph.D. degree in electrical engineering from Northwestern University, Evanston, IL, in 1993 and 1998, respectively.

She is currently an Assistant Professor with the Department of Electrical and Computer Engineering, University of Wisconsin (UW)-Madison. She is also a faculty affiliate of the Department of Biomedical Engineering and a member of the UW Comprehensive Cancer Center. Her research interests include FDTD theory and applications, electromagnetic imaging, sensing, and exposure techniques for biological and medical applications, and full-wave analysis and design of photonic microstructures. She was named one of the 100 top young innovators in science and engineering in the world by the Massachusetts Institute of Technology's Technology Review magazine in 2002.

Dr. Hagness is currently an associate editor for the IEEE ANTENNAS AND Wireless PROPAGATION LETTERS. She is a member of the IEEE Antennas and Propagation Society (IEEE AP-S) Administrative Committee (AdCom). She is also the membership chair for Commission B of the U.S. National Committee (USNC)/URSI and the IEEE AP-S representative to the IEEE-USA Medical Technology Policy Committee. She was the recipient of the 2000 Presidential Early Career Award for Scientists and Engineers (PECASE) from the White House and the 2002 Booker Fellowship Award presented by the USNC/URSI. She was also the recipient of several teaching awards, including the 2003 UW Emil Streiger Distinguished Teaching Award.

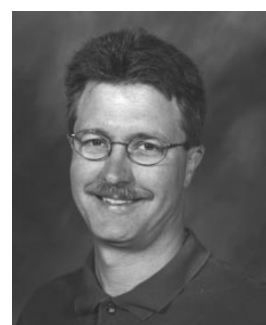

John H. Booske (S'82-M'85-SM'93) received the $\mathrm{Ph} . \mathrm{D}$. degree in nuclear engineering from The University of Michigan at Ann Arbor, in 1985.

From 1985 to 1989 , he was a Research Scientist with the University of Maryland at College Park. In 1990, he joined the faculty of the University of Wisconsin-Madison, where he is currently a member of the Department of Electrical and Computer Engineering and Director of the Interdisciplinary Graduate Materials Science Program. His research interests include the experimental and theoretical study of coherent electromagnetic radiation sources and their applications, with emphasis in the RF, microwave, and terahertz regimes. His recent research activities include vacuum electronics, microwave and RF heating of materials, plasma processing of materials, and biomedical applications of microwave and RF fields.

Prof. Booske was the recipient of the U.S. National Science Foundation Presidential Young Investigator Award. He has also been the recipient of numerous teaching awards, including the University of Wisconsin Chancellor's Distinguished Teaching Award and the Benjamin Smith Reynolds Award for Excellence in Teaching Engineering.

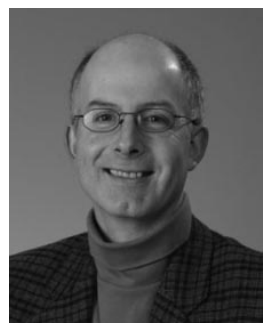

Michal Okoniewski (S'88-M'89-SM'97) received the M.S.E.E. and Ph.D. (with distinctions) degrees from the Technical University of Gdansk, Gdansk, Poland, in 1984 and 1990, respectively.

From 1984 to 1986, he was with the Institute of Fluid Flow Machinery, Polish Academy of Sciences. From 1986 to mid-1992, he was with the Technical University of Gdansk, as an Assistant Professor. In 1992, he joined the University of Victoria, Victoria, BC, Canada, as a Natural Sciences and Engineering Research Council of Canada (NSERC) International Post-Doctoral Fellow and later as a Research Fellow and Adjunct Professor. Since 1998, he has been an Associate Professor with the Department of Electrical Engineering, University of Calgary, Calgary, AB, Canada, and a Scientist with TR-Labs Calgary, Calgary, AB, Canada. In 2002, he was appointed a prestigious Canadian Research Council (CRC) Chair position in applied electromagnetics. His current research interests include applied and computational electromagnetics, antennas, passive components, RF microelectromechanical systems (MEMS), microwave photonics, dielectric spectroscopy, and custom computational hardware.

Dr. Okoniewski is an associate editor of the IEEE TRANSACTIONS ON ANTENNAS AND PROPAGATION. 